\title{
A Partial-Current-Steering Biphasic Stimulation Driver for Vestibular Prostheses
}

\author{
Timothy G. Constandinou, Member, IEEE, Julius Georgiou, Member, IEEE, and Christofer Toumazou, Fellow, IEEE
}

\begin{abstract}
This paper describes a novel partial-current-steering stimulation circuit for implantable vestibular prostheses. The drive hardware momentarily delivers a charge-balanced asymmetric stimulus to a dummy load before steering towards the stimulation electrodes. In this fashion, power is conserved while still gaining from the benefits of current steering. The circuit has been designed to be digitally programmable as part of an implantable vestibular prosthesis. The hardware has been implemented in AMS $0.35 \mu \mathrm{m}$ 2P4M CMOS technology.
\end{abstract}

Index Terms-Artificial electrical stimulation, biphasic, bipolar, continuous interleave sampling, current-steering, neural stimulation, neuroprosthetic, vestibular prosthesis.

\section{INTRODUCTION}

A RTIFICIAL electrical stimulation, a methodology becoming increasingly accepted in the medical community, is providing engineers and medical professionals a reliable method to interface to neural tissue. Neural prostheses are already benefitting those with profound hearing loss, cardiac arrythmia, loss of muscular function, hand grasp, foot drop, bladder control, and soon those with loss of vision. A key component in such systems is the neural interface and stimulation drive hardware. Reliability and robustness are paramount, and ensuring good efficiency and minimizing neural fatigue ensures long-lasting rehabilitation.

Restoring the sensation of inertia in individuals with balancerelated impairments is achievable through development of an artificial vestibular prosthesis, based on the cochlear implant paradigm. The inner ear's vestibular system provides cues about self-motion and help stabilize vision during movement. Damage to this system can result in dizziness, imbalance, blurred vision, and instability in locomotion, a leading cause of death in the elderly. Restoration of balance can, therefore, be achieved by bypassing a dysfunctional element in the vestibular pathway using artificial stimulation. A vestibular prosthesis would incorporate

Manuscript received January 15, 2008; revised March 12, 2008. First published July 25, 2008; current version published September 10, 2008. This work was supported by the Cyprus Research Promotion Foundation (RPF) under Grant $\Pi \Delta \mathrm{E}-0505 / 07$. This paper was recommended by Associate Editor B.-D. Liu.

T. G. Constandinou is with the Institute of Biomedical Engineering at Imperial College London, London SW7 2AZ, U.K., and also with the Holistic Electronics Research Laboratory, University of Cyprus, Nicosia 1678, Cyprus (e-mail: t.constandinou@imperial.ac.uk).

J. Georgiou is with the Holistic Electronics Research Laboratory, Department of Electrical and Computer Engineering, University of Cyprus, Nicosia 1678, Cyprus (e-mail: julio@ucy.ac.cy).

C. Toumazou is with the Institute of Biomedical Engineering, Imperial College London, London SW7 2AZ, U.K. (e-mail: c.toumazou@imperial.ac.uk).

Color versions of one or more of the figures in this paper are available online at http://ieeexplore.ieee.org.

Digital Object Identifier 10.1109/TBCAS.2008.927238 miniature (MEMS-based) inertia sensors, suitable sensor interfacing circuits, a semicircular canal processor and stimulation driver. Research in developing artificial vestibular prostheses has recently gained much momentum, with a number of groups [1]-[3] implementing discrete electronics for inertia sensing, processing, and neural stimulation.

This work aims to implement an integrated hardware solution towards a single chip semicircular canal stimulator based on techniques used within cochlear prosthetics. This paper presents a novel CMOS-based integrated circuit for artificial neural stimulation as part of an implantable vestibular prosthesis. The paper is organized as follows-Section II provides some theoretical background regarding stimulation parameters, while Section III outlines the system architecture. Section IV describes the implementation at circuit level and Section V presents the measured results. Finally, Section VI summarizes and discusses the various features of the presented work.

\section{STIMULATION THEORY}

Typically, in electrical neural stimulation, a minimum of two electrodes are used to produce a nerve activation current. The pair can be used in a monopolar (shared reference electrode) or a bipolar (individual electrode pairs) configuration [4]. For applications requiring greater activation selectivity, the bipolar scheme is preferred because each pair generates a more localized field (compared to monopolar) [5], [6]. However, bipolar stimulation schemes typically consume higher power due to increased shunting currents. Stimulus pulse parameters include frequency, amplitude and duration. The former affects the smoothness of perceived sensation and needs to be adjusted to prevent neural fatigue. The latter two parameters affect the strength of the neural response and alter the charge injected. Another issue to consider is the stimulus drive mechanism, i.e., by what means the stimulus is delivered to the target site. The three methods are: voltage-mode, current-mode, and charge-mode [7]. Although different systems may be based on different mechanisms, we consider this to be an implementation issue; as the underlying goal is to deliver (and then remove) a well-defined charge packet.

As established by [8], in order to avoid harmful electrochemical processes, the stimulus waveform has to be biphasic. In such a waveform the first pulse causes activation, followed by a second one with opposite polarity to balance the charge delivered by the first [9]. However, it has been reported [10] that two opposite pulses back to back could act to prevent the generation of an action potential, or could require more energy to produce an action potential. To overcome this, a short time delay needs to be introduced between the pulses. This delay allows the action 
potential to propagate away from the stimulation site before the electrode recovers the injected charge. Additionally, using an extended anodic pulse with reduced amplitude can compensate for charge distribution and thus reduce fatigue. The various aspects of the waveform profile are discussed in [11].

\section{SYSTEM OVERVIEW}

This system implements a 3-channel biphasic current-mode stimulation drive with digitally programmable signal conditioning for a fully-implantable vestibular prosthesis [12] using a bipolar electrode configuration. Due to the close proximity between adjacent stimulation sites, the system incorporates a continuous interleave sampling (CIS) strategy to minimize crosstalk. This strategy involves sequencing the stimulation waveforms between the different channels such that only one is active at any given time. Furthermore, we have chosen to implement an asymmetric (but charge-balanced biphasic) waveform profile, to match natural neurophysiological response, thus reducing fatigue of neural tissue. Although in general, CIS stimulation strategy utilizes symmetrical stimulation pulses, with same duration of positive and negative pulses, we have chosen to utilize an asymmetrical stimulation pulses with 1:4 ratio. This implementation is limited in that fewer channels can be utilized for interleaved stimulation, however, in the case of the vestibular prosthesis, the maximum number of required channels is limited to just 5, i.e. at the nerve endings of the three semicircular canals and two otolith organs.

It should be mentioned that although extensive multipurpose stimulators [13]-[21] have been developed, we have elected to implement a simpler variant as the vestibular implant requires to incorporate additional hardware (including MEMS and electronics) in as small as possible space. For the purposes of this application, the telemetry link is not operated continually to stream the stimulation data onto the device (self-motion information is generated on chip) but rather in a one-off cycle simply to programme the stimulation channel characteristics.

The chosen charge-delivery methodology is current-mode, as this is the least effected by stimulation target impedance variation. Additionally, the system includes a short-duration current steering phase, to avoid charge-buildup, and therefore glitching at turn-on and turn-off, while reducing power consumption compared to traditional current-steering systems [22]. A study [23] recently reported that using current steering techniques can additionally be considered for increased spectral resolution. Finally, as an added precaution, once each CIS cycle, all the stimulation electrodes are grounded, such that any residual charge that may have accumulated (due to device mismatch, electrode variation, etc.) is removed, although this is expected to be at least three orders of magnitude lower than the stimulus.

The target stimulation profile and current-steering methodology used is illustrated in Fig. 1. For one clock cycle before and after each stimulation phase, the current is steered towards a dummy load, such to achieve a smooth transition, thus minimizing charge buildup and spiking. Additionally, the current magnitude is scaled with ratio $4: 1$ between cathodic and anodic pulses. Conversely, the pulse lengths are scaled 1:4, respectively, to maintain charge balance. The corresponding current
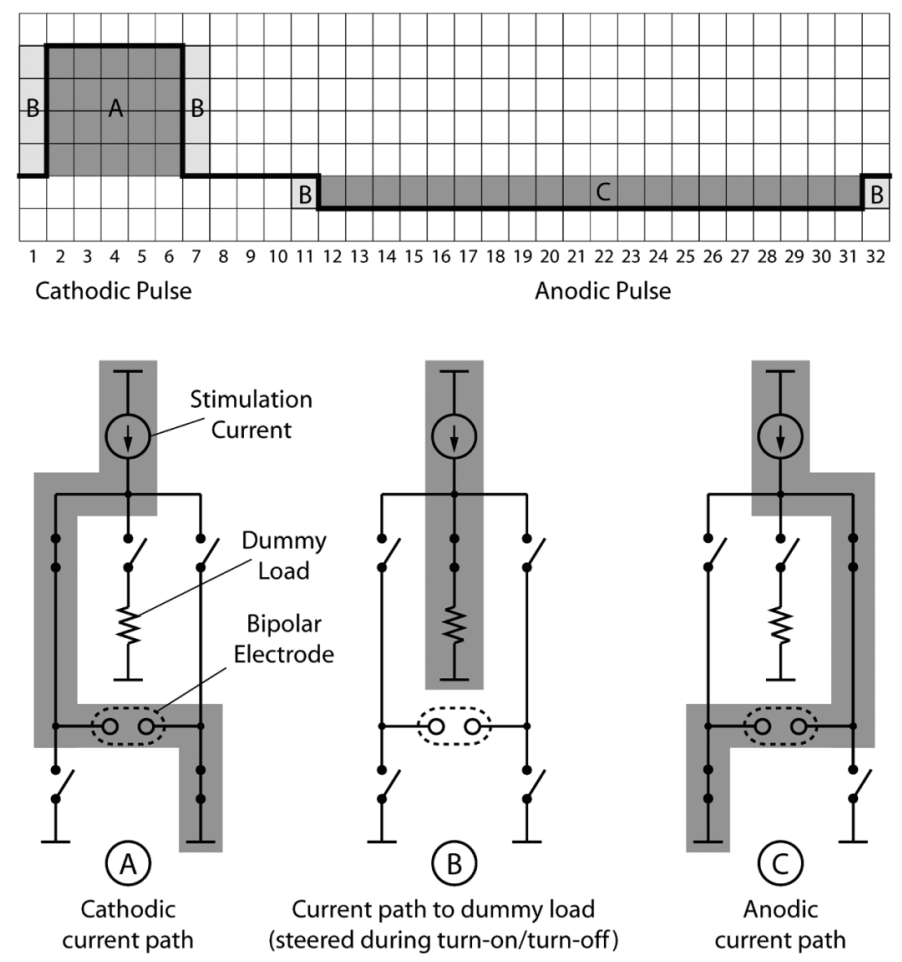

Fig. 1. Stimulation profile (top) with annotated current-path for corresponding phases.

paths flowing through the drive switches are also shown (circuit details are given later). Another feature is that there exists a short pause between cathodic and anodic pulses.

\section{Circuit ImPlementation}

The top-level system schematic is shown in Fig. 2. This shows the 3-channel stimulation drive incorporating a CIS strategy [24], based on a modular design architecture, similar to [25]. The implementation and purpose of the various sub-blocks will be discussed in the following subsections.

\section{A. Stimulus Conditioning Circuit}

The stimulation current levels required for a patient to just about perceive a sensation (stimulation threshold) varies quite significantly from patient to patient or even from electrode site to electrode site within the same end-organ, in our case the vestibule. This greatly depends on the number of surviving neurons and the proximity of the electrodes to the nerves. Similarly, the maximum comfortable stimulation level also has a large variability. However, in all cases, the dynamic range between the minimum threshold and the maximum comfortable level is quite low, for example, in the cochlear ranging typically from 6 to $20 \mathrm{~dB}$. Hence, good fitting is important if the most is to be made of the limited dynamic range of the patient. Consequently, for each stimulation channel, digital settings have been included for programmability [19], [25], [26] of: 1) offset (3-bit); 2) gain (4-bit); and 3) threshold (3-bit). These are intended to: 1) remove any static (operating point) offset caused by process variation and/or device mismatch in preceding hardware; 2) amplify the input stimulus such that the dynamic range is maximized for 


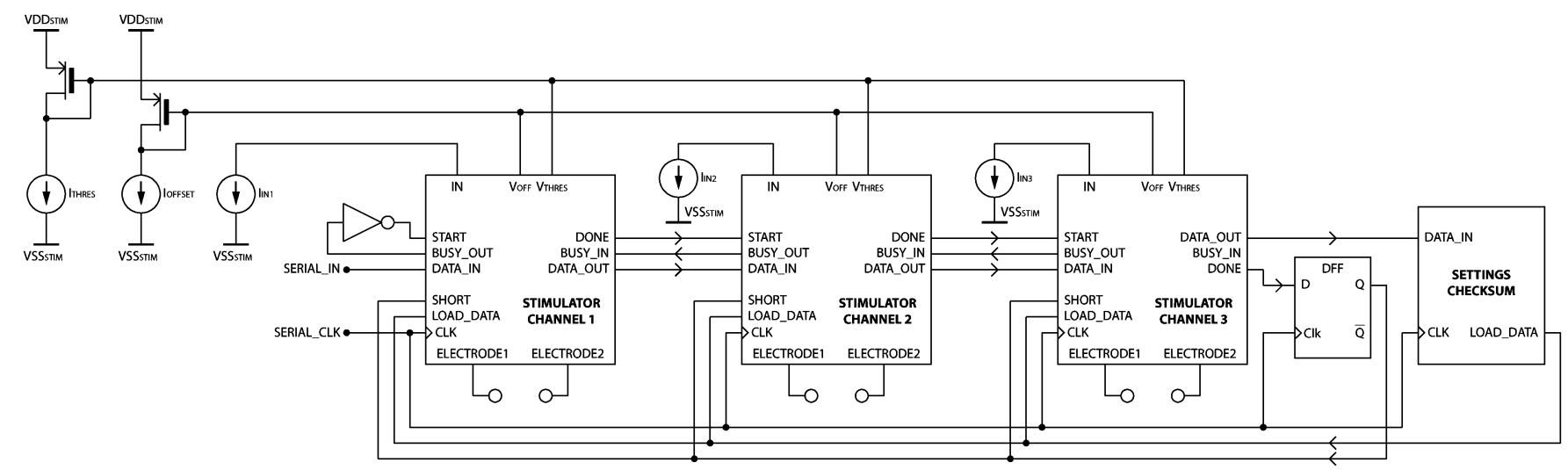

Fig. 2. Top level circuit schematic for the 3-channel electrical stimulation circuit. The modular scheme allows for additional channels to be easily incorporated within the CIS strategy.

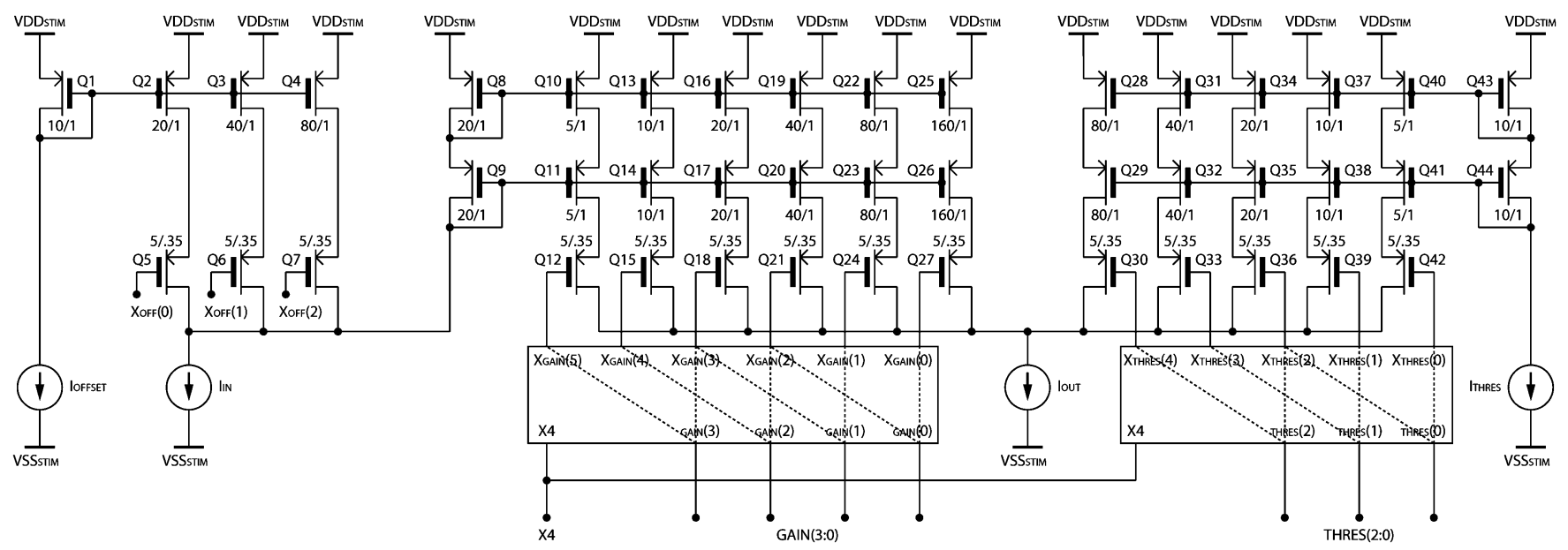

Fig. 3. Current-mode mirror network to condition the stimulation current. Programmability includes 3-bit offset compensation (for tuning out mismatch-related offset), 4-bit gain control and 3-bit stimulus threshold (for fitting patients threshold of sensation). Additionally, two input multiplexers are required to further scale $(\times 4)$ the threshold and gain during the cathodic phase to produce a correctly scaled asymmetric waveform. This is implemented for each stimulation channel. All devices shown are high- $V_{T}$ (thick oxide).

a particular channel/patient; and 3) provide the baseline stimulation to match the onset of sensation of the patient. The fitting circuits consist of digitally controlled variable-width current mirrors, as shown in Fig. 3. The current mirror network produces a stimulation current given by

$$
I_{C A}=4 \cdot I_{A N}=M_{T} \cdot I_{T}+M_{G} \cdot\left(I_{I N}-M_{O} \cdot I_{O}\right)
$$

where $I_{C A}$ and $I_{A N}$ are the cathodic and anodic current magnitudes, $I_{I N}$ is the input current, $I_{T}$ and $I_{O}$ are the threshold and offset bias currents (corresponding to $1 \mathrm{LSB}$ ) and $M_{T}, M_{O}$, and $M_{G}$ are the threshold (0-7), offset (0-7), and gain (0-15) discrete (digital) multipliers. Additionally, during the cathodic phase, both the threshold and gain inputs (digital) are shifted 2-bits to the left, thus scaling the currents $(\times 4)$.

For a stimulus (biphasic pulse) period of 32 clock cycles, the charge injected (i.e. for 5 clock cycles at $4 \times$ amplitude) and removed (i.e. for 20 clock cycles at $1 \times$ amplitude) during each stimulation phase is given by

$$
Q=\frac{20}{f_{C l k}} \cdot I_{A N}
$$

where $Q$ is the stimulus charge and $f_{C l k}$ is the clock frequency.
Cycling this stimulus at the CIS refresh rate, gives the following net charge transfer rate:

$$
Q / t=\frac{\frac{20}{f_{C l k}} \cdot I_{A N}}{\frac{32}{f_{C l k}} \cdot N_{C H}+1}
$$

where $N_{C H}$ is the number of CIS stimulation channels and the +1 term is to include the SHORT phase.

\section{B. Patient Data Loading Circuit}

In order to implement a feasible totally-implantable prosthesis, the requirement for digitally programmability is paramount. One challenge in achieving such functionality is the scheme used for data transmission and recovery. Typically, medical implants use inductive schemes whereby patient settings data is encoded onto a carrier signal that is inductively coupled through the skin, to the subcutaneously implanted device where the data can be recovered [27], [28]. Implementing robust hardware to extract patient settings data from a continuous bitstream requires: 1 ) a temporary settings (shift) register and method to precisely align the incoming bitstream to patient settings register; 2) a method to reliably initiate a parallel load from serial input to patient settings register; and 3) data 


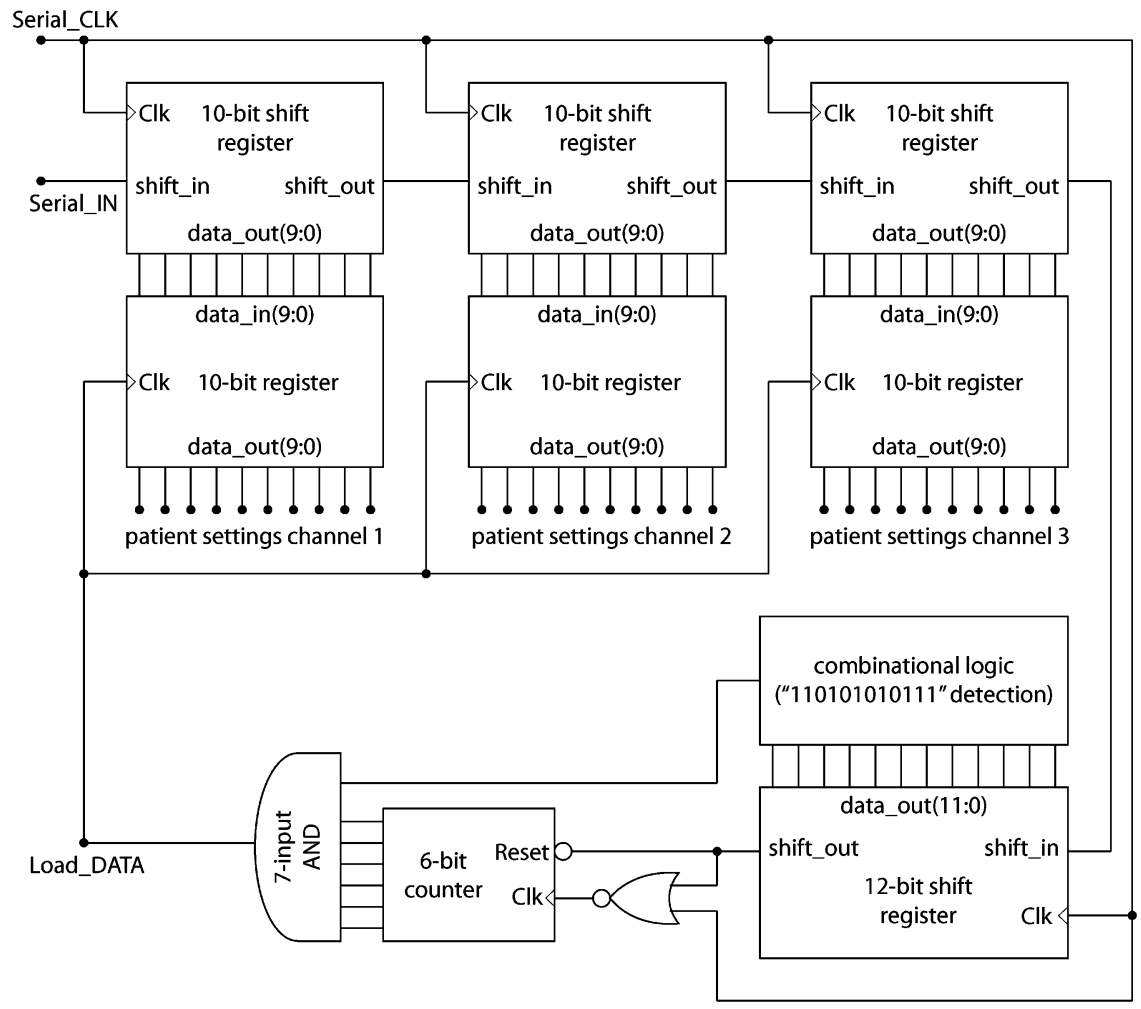

Fig. 4. State machine for loading and storing patient settings using a single bitstream (to be recovered from inductive telemetry).

redundancy and error correction. The design implemented in this system is shown in Fig. 4 (adopted from [25]). This work however, does not include hardware for data redundancy and error correction. It is envisaged that this can be implemented within the data recovery circuits using standard coding techniques, eg. hamming coding, parity check-bits, etc.

The hardware for loading the patient tuning data can be divided into two sections; the registers that are specific to each channel, and the state machine for determining when a parallel-register-load occurs. This is illustrated in Fig. 4. The input serial data stream passes through a serial shift register into the state machine to check for the start sequence. Once the start sequence is detected, this signifies the data within the serial registers are perfectly aligned with the patient settings register and a parallel register load can occur. These input and patient registers are repeated (and cascaded) for each channel, i.e. within a 3-channel system, each register would be of $3 \times[3-$ bit(offset) $+4-$ bit(gain) $+3-$ bit(threshold)] $=30-$ bit length. The state machine, appended to the end of the combined shift-register interrogates the incoming bitstream to match a multi-bit START-SEQUENCE chosen to be: 111010101011 . Finally, a ripple counter increments for every " 0 " received, and reset whenever a " 1 " is received, thus checking for the occurrence of 64 successive 0 's. The LOAD-DATA signal is therefore asserted when both these conditions are met, i.e. the patient settings bitstream starts with 64 0's followed by the START-SEQUENCE. The purpose of the 640 's is to flush the serial shift register prior to loading the patient settings, thus to ensure a partial data set cannot trigger a parallel register load.

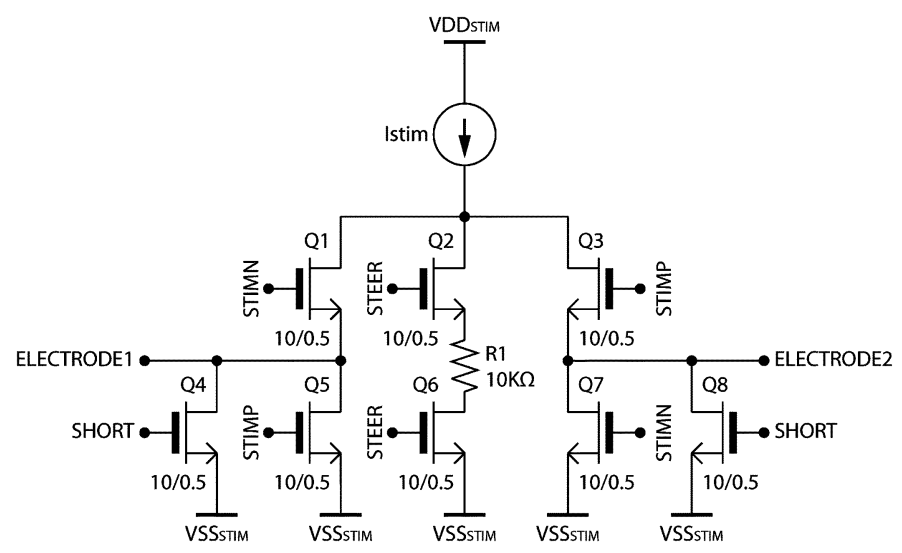

Fig. 5. Circuit schematic for H-bridge with current steering (to/from dummy load) during turn-on/turn-off transitions. Dummy load (R1) is chosen to match that of the stimulation electrodes and neural tissue $(\approx 10 \mathrm{k} \Omega)$.

\section{CIS Biphasic Waveform Generation Circuit}

The CIS generator is the last of the signal conditioning blocks that directly interfaces with the electrodes, via blocking capacitors. The CIS generator converts the output of the patient dynamic range mapping circuits into non-overlapping biphasic pulses. A top-level block diagram of the CIS generator has been shown in Fig. 2. As there are three channels in the illustrated system, there are three output driver cells making up the CIS generator. Each output driver cell includes a biphasic waveform generation circuit, each of which includes a serial shift register such that the stimulation control propagates down the cascade. At the end of the chain (i.e. after the last channel) 
an extra flip-flop has been added so that it can provide an extra pulse that shorts all electrodes to ground, as to remove any residual charge. This is required to make absolutely sure that no DC charge accumulates on the blocking capacitors, reducing voltage compliance. Alternatively, techniques are being developed [29]-[32] to avoid requiring blocking capacitors, for example, using charge metering [20], [30], feedback DAC calibration [31] and voltage monitoring [21], [32].

The biphasic waveform (shown previously in Fig. 1) is generated using a state machine; requiring a clock $32 \times$ higher than the CIS clock. The state machine is based on a 32-bit serial shift register, passing a single-bit token to trigger the various events, using RS-latches specifically arranged to capture the individual phases. For example, the anodic phase has been predefined to start on clock cycle 12 and end after clock cycle 31 . All the bits from within this serial shift register are NOR'ed and the result is fed round the loop to the input. This technique guarantees robustness as any extra active bits in the register are flushed out after the first cycle. Beyond that the circuit can only have one active flip-flop as it will only generate a new token once there are none in the register.

These digital control signals generated above (after being level-shifted to stimulation supply voltage), feed the current control switches to form the H-bridge, shown in Fig. 5, the functioning of which has been previously illustrated in Fig. 1. In this, Q1 and Q7 drive the cathodic pulse, devices Q3 and Q5 drive the anodic pulse, devices Q2 and Q6 drive the dummy load (steering), and devices Q4 and Q8 are used to ground the stimulation electrodes, during the short/reset phase.

\section{Integrated Circuit}

The prototype chip was fabricated in AMS $0.35 \mu \mathrm{m} 2 \mathrm{P} 4 \mathrm{M}$ CMOS technology with a die size of $1.5 \mathrm{~mm} \times 0.9 \mathrm{~mm}$. A microphotograph and floorplan of the complete chip is shown in Fig. 6.

The padring has been organized into two sections, a $3.3-\mathrm{V}$ digital section for telemetry inputs (5 bondpads at top) and a $10-\mathrm{V}$ analog section (using thick oxide devices) for the current-mode inputs and stimulation outputs (15 bondpads along sides and bottom). Although the power saved in having a lower voltage logic supply is negligible (together with the added complication of level shifting), this was chosen such that when the battery supply is low, all the other supplies are cut off to prevent complete discharge. Furthermore, in the event that the registry power is completely cut off, on power up the system resets the registry's contents to ensure that the system comes up in a safe state, i.e., all outputs are set to zero.

\section{MEASUREd RESUlts}

The circuit was biased using off-chip current sources (Keithley models 2602 and 6221) on a custom PCB test platform. Bias currents are set to $I_{\mathrm{LSB}-\mathrm{THRESHOLD}}=15 \mu \mathrm{A}$ and $I_{\mathrm{LSB}-\mathrm{OFFSET}}=150 \mathrm{nA}$, allowing for an input current range of $0-10 \mu \mathrm{A}$ to provide the output within the desired range.
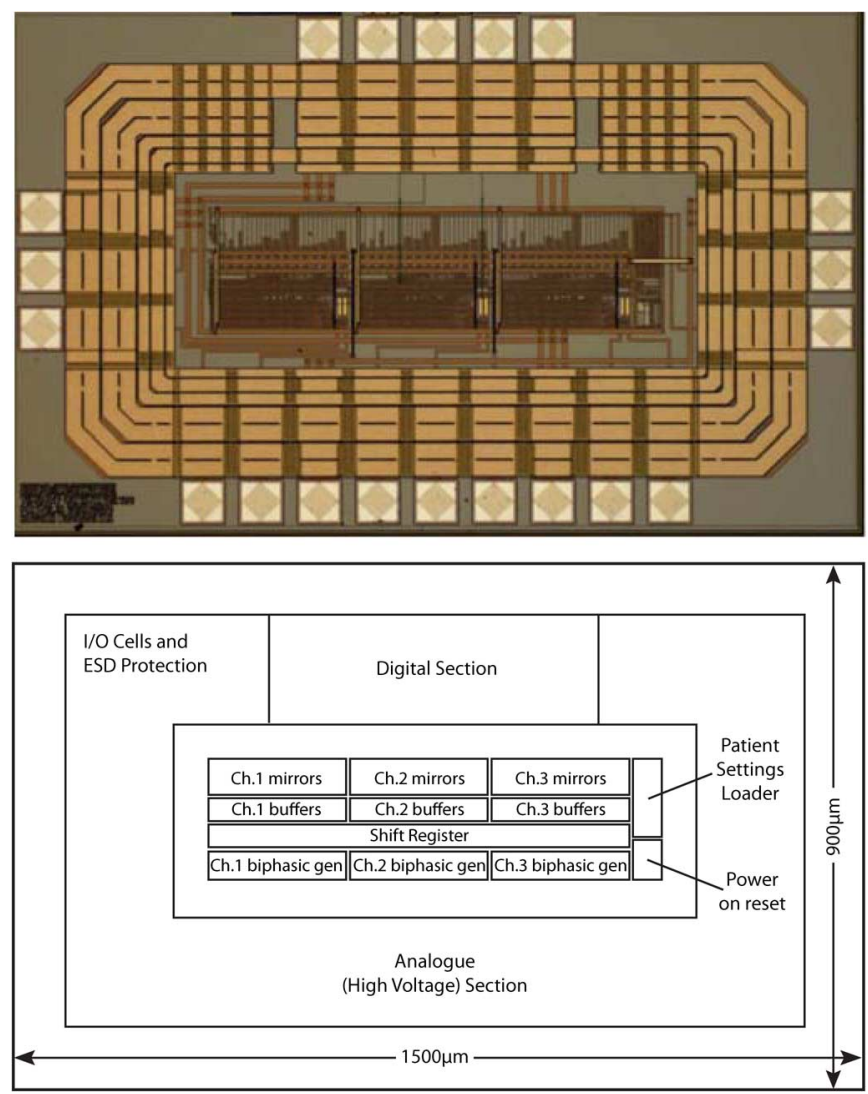

Fig. 6. Chip microphotograph and floorplan for the 3-channel stimulation circuit.

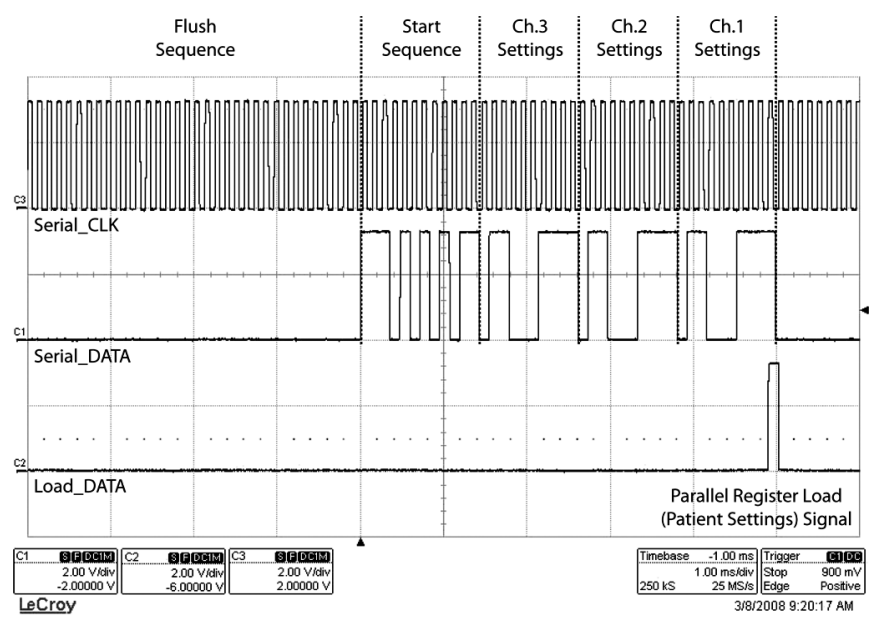

Fig. 7. Measured response of the LOAD-DATA signal on an input serial data stream. In the example, the patient settings data on all channels have been selected to have maximum gain and threshold and zero offset correction.

\section{A. Loading Patient Settings}

The patient settings, i.e. serial bitstream and clock is generated (in the format previously described in Section IV-B) using an off-the-shelf microcontroller. This confirms the correct operation of the state machine, illustrated in Fig. 7, where it can be seen that the circuit issues a LOAD-DATA signal after complete transmission of the settings data. 


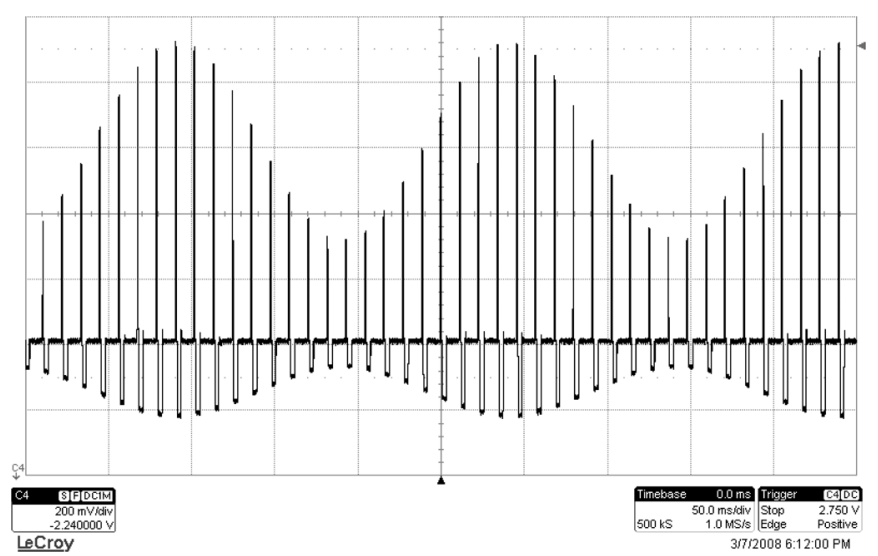

Fig. 10. Measured stimulation profile of a channel with a 5-Hz sinusoidal input stimulus.

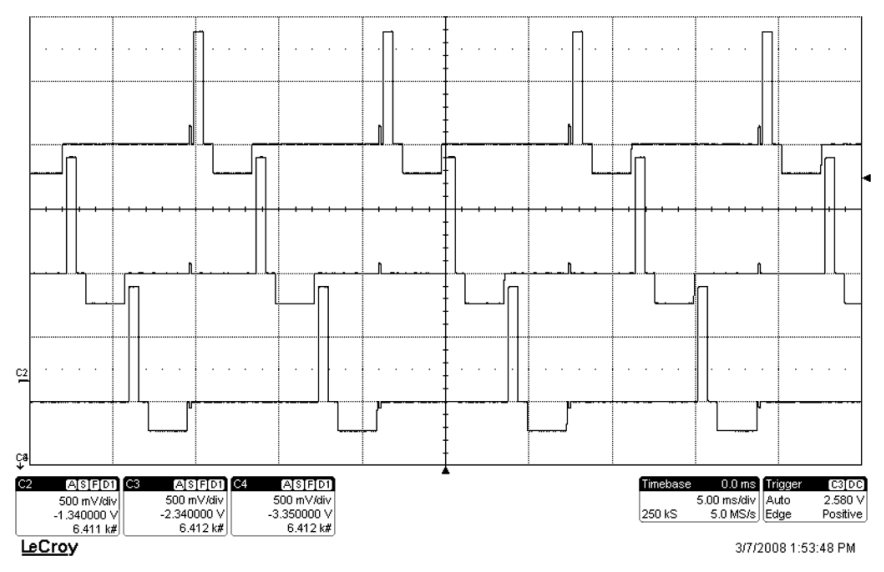

Fig. 8. Measured stimulation output of the three channels illustrating the CIS sequencing and biphasic (asymmetric, charge-balanced) waveform profile.
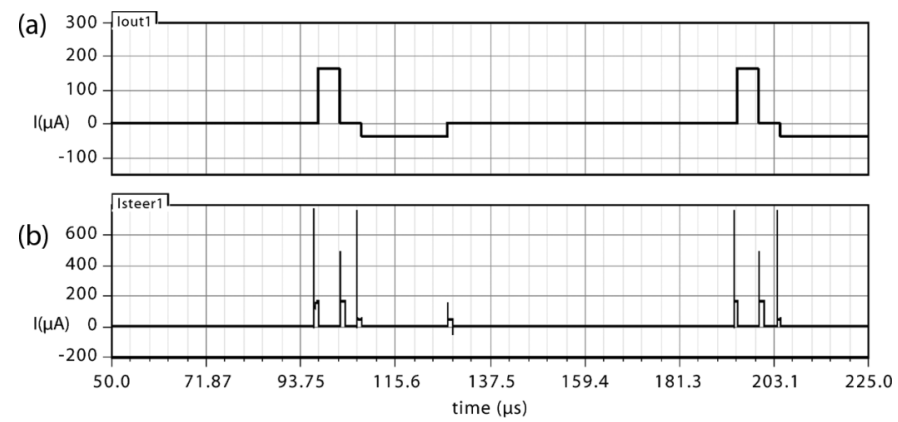

Fig. 9. Transient simulation results illustrating the current-steering technique. Shown are: (a) stimulation current delivered to electrode and (b) current steered through the dummy load.

\section{B. Stimulation Output}

Measurements are taken of the circuit's output current delivered to a $10 \mathrm{k} \Omega$ load through a $100 \mathrm{nF}$ blocking capacitor. This effectively resembles a high-pass response with $f_{3 \mathrm{~dB}}=159 \mathrm{~Hz}$. The measured stimulation outputs (at maximum threshold with zero input) are shown in Fig. 8. The three biphasic waveforms are sequenced to the intended CIS strategy and the SHORT pulse (leading each CIS cycle) illustrates the removal of any residual charge. Furthermore, the

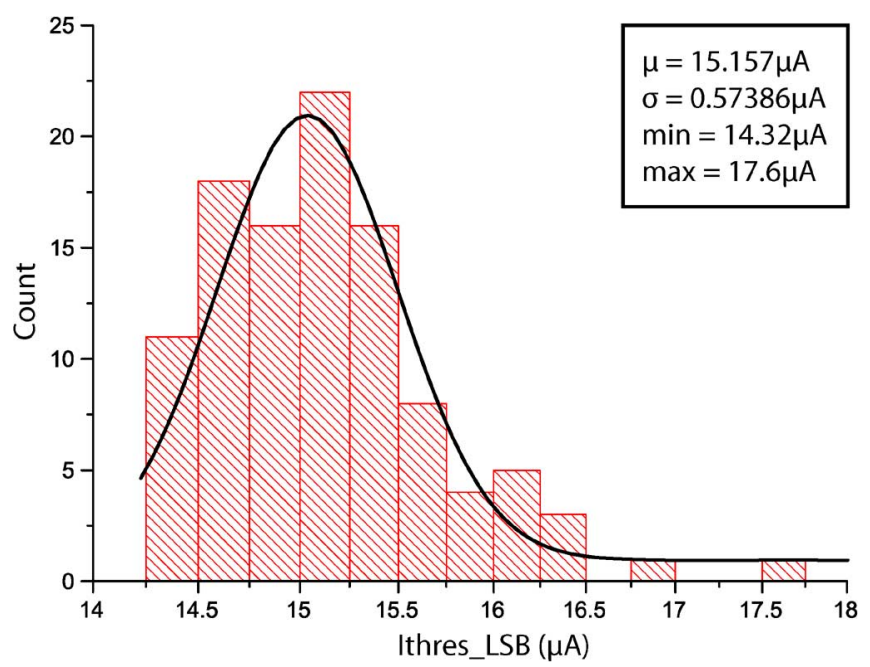

Fig. 11. Measured variation in threshold linearity for $I_{\text {thres }}=15 \mu \mathrm{A}$ normalized to $1 \mathrm{LSB}$.

TABLE I

TARGET DESIGN SPECIFICATIONS

\begin{tabular}{ll}
\hline CMOS Technology & AMS 0.35 $\mu \mathrm{m}$ 2P4M CMOS \\
Supply Voltage & $3.3 \mathrm{~V}$ (logic), 10V (stimulation) \\
$\begin{array}{l}\text { Die Area } \\
\text { Device Count } \\
\text { Number of Channels }\end{array}$ & 6946 \\
\hline Stimulation Strategy & 3 \\
Stimulation Pulse Type & Continuous Interleave Sampling \\
\hline Threshold Resolution & 3-bit (Max. 105 $\mu \mathrm{A}$ ) \\
Gain Resolution & 4-bit $(\mathrm{Max} .630 \mu \mathrm{A}$ ) \\
Stimulation Current & 0 to $735 \mu \mathrm{A}$ \\
Pulse Width & 25 to $100 \mu \mathrm{s} / \mathrm{phase} *$ \\
\hline Static Current Consumption & $19.847 \mu \mathrm{A} \dagger$ \\
\hline
\end{tabular}

$*$ By tuning system clock, †Excluding stimulation drive

clean turn-on/turn-off transitions show that the chosen (partial) current-steering approach is operating as intended.

The internal operation of the current steering to overcome the turn-on and turn-off glitches can be illustrated through the simulation results, shown in Fig. 9. This clearly shows how the glitches are dissipated within the on-chip dummy loads.

The measured stimulation waveform for a sinusoidal $(5 \mathrm{~Hz})$ input current of $10 \mu \mathrm{A}$ (ptp) is shown in Fig. 10. This illustrated the effect of the threshold signal to offset the input stimulation signal.

\section{Process Variation}

At design time, statistical Monte Carlo simulations had been performed to ensure monotonicity in the patient settings, i.e., so that any inaccuracies in current magnitudes due to technology/ process/mismatch variations remain below $1 / 2$ LSB. For this, the current mirror device sizes have been selected such that a $1 / 2$ LSB current variation corresponds to $>3 \sigma$. The measured nonlinearity in threshold stimulus (i.e., variation in $1 \mathrm{LSB}$ ) is in agreement with the intended design specifications, shown in Fig. 11. The measured current variation, normalized to $1 \mathrm{LSB}$ 
TABLE II

COMPARISON WITH OTHER WORK

\begin{tabular}{|c|c|c|c|c|c|}
\hline Reference & [15] & [16] & [18] & {$[20]$} & This work \\
\hline Year & 1999 & 1999 & 2005 & 2007 & 2008 \\
\hline Technology & Mietec $2 \mu \mathrm{m}$ & $\mathrm{NTE} 1.2 \mu \mathrm{m}$ & TSMC $0.35 \mu \mathrm{m}$ & $0.18 \mu \mathrm{m}$ & AMS $0.35 \mu \mathrm{m}$ \\
\hline Supply & $12 \mathrm{~V}$ & 1999 & $3.3 \mathrm{~V}$ & $3.3 \mathrm{~V}$ & $3.3 / 10 \mathrm{~V}$ \\
\hline Die Area & $3 \times 4 \mathrm{~mm}$ & $16 \mathrm{~mm}^{2}$ & $1.98 \times 2.17 \mathrm{~mm}$ & $3.2 \times 2.8 \mathrm{~mm}$ & $1.5 \times 0.9 \mathrm{~mm}$ \\
\hline Channels & 4 & 4 & 2 & $4 \times 4$ (per unit) & 3 \\
\hline Output Range & $2 \mathrm{~mA}$ & $0.7-6.3 \mathrm{~mA}$ range $^{1}$ & $1.75 \mathrm{~mA}$ & $18-140 \mu \mathrm{A}$ range $^{1}$ & $0-735 \mu \mathrm{A}$ range $^{2}$ \\
\hline Signal Resolution & 8-bit & 6-bit & 5-bit & 5-bit & current input, $48 \mathrm{~dB}^{3}$ \\
\hline Time Resolution & $255 \mu \mathrm{s}$ & $10-2550 \mu \mathrm{s}$ & $0.1-2 \mathrm{~ms}$ & variable (per channel) & $25-100 \mu \mathrm{s}$ \\
\hline Stimulus Type & bipolar & bipolar & bipolar & bi- or monopolar & bipolar \\
\hline Stimulus Waveform & asymm. biphasic & arbitrary & symm. mono/bi-phasic & symm. biphasic & asymm. biphasic \\
\hline Stimulus Mismatch & $25 \mathrm{nC}(5 \%)$ & - & - & $2 \%$ & $0.5 \%$ \\
\hline Charge Balancing & 4.7nF block. cap. & - & - & $\mathrm{I} / \mathrm{V}$ monitoring ${ }^{4}$ & 100nF block. cap. \\
\hline
\end{tabular}

${ }^{1}$ 2-bit per ch. range selection, ${ }^{2}$ 10-bit per ch. dynamic range (DR) mapping, ${ }^{3}$ minimum DR, ${ }^{4}$ 8-bit stimulation I/V monitoring/compensation

ranged from 14.32 to $17.6 \mu \mathrm{A}$, tested on all 3 channels over 10 dies.

\section{Power Consumption}

The power consumption is largely dominated by the stimulation supply and thus largely dependant on patient settings. The total static current consumption has been measured to be $19.847 \mu \mathrm{A}$ (for $I_{\text {thres }}=15 \mu \mathrm{A}$ and $I_{\text {offset }}=150 \mathrm{nA}$ ). This consists of a $4.667-\mu \mathrm{A}$ portion consumed by the logic supply and $15.15 \mu \mathrm{A}$ by the analog supply (in biasing the current mirrors).

\section{E. System Specifications}

These are given in Table I.

\section{CONCLUSION}

In this paper, we have presented the an integrated circuit for artificial electrical stimulation of neural tissue within the inner ear for stimulation of the vestibular organ.

This system includes three (modular) stimulation channels, each with full digital programmability, sequenced using the CIS stimulation strategy and can be easily expanded to include more channels. We have focused on achieving good reliability and robustness, specifically to ensure long-term stability, be minimally invasive and guaranteeing that patient calibration settings are uploaded in a safe manner. To this goal, we have implemented a novel current-steering scheme to avoid charge-buildup-related stimulation artifacts. This has designed to ensure all stimulation transitions are smooth but sharp while maintaining good power efficiency, compared to traditional current-steer systems. Moreover, the system has been designed to generate an asymmetric, charge-balanced waveform to maximize neural response to electrical stimulus whilst minimizing fatigue of neural tissue. A comparison of this work with other neural stimulator chips is listed in Table II.

\section{REFERENCES}

[1] W. Gong and D. Merfeld, "A prototype neural semicircular canal prosthesis using patterned electrical stimulation," Ann. Biomed. Eng., vol. 28 , pp. $572-581,2000$
[2] A. M. Shkel and F.-G. Zeng, "An electronic prosthesis mimicking the dynamic vestibular function," Audiol. Neurotol., vol. 11, pp. 113-122, 2006.

[3] C. C. Della Santina, A. A. Migliaccio, and A. H. Patel, "A multichannel semicircular canal neural prosthesis using electrical stimulation to restore 3-D vestibular sensation," IEEE Trans. Biomed. Eng., vol. 54, no. 6, pp. 1016-1030, Jun. 2007.

[4] P. H. Peckham and J. S. Knuston, "Functional electrical stimulation for neuromuscular applications," Ann. Rev. Biomed. Eng., no. 3, pp. 327-360, 2005.

[5] P. Comte, "Monopolar versus bipolar stimulation," Stereotactic and Functional Neurosurgery, vol. 45, no. 1-2, pp. 156-159, 1982.

[6] B. J. Kwon and C. Van den Honert, "Effect of electrode configuration on psychophysical forward masking in cochlear implant listeners," $J$. Acoust.Soc. Amer., vol. 119, no. 5, pp. 2994-3002, 2006.

[7] J. Simpson and M. Ghovanloo, "An experimental study of voltage, current, and charge controlled stimulation front-end circuitry," in Proc. IEEE Int. Symp. on Circuits Syst., 2007, pp. 325-328.

[8] J. C. Lilly, J. R. Hughes, E. C. Alvord, and T. W. Galkin, "Brief, noninjurious electric waveform stimulation of the brain," Science, vol. 121, no. 3144, pp. 468-469, 1955.

[9] J. T. Rubinstein, C. A. Miller, H. Mino, and P. J. Abbas, "Analysis of monophasic and biphasic electrical stimulation of nerve," IEEE Trans. Biomed. Eng., vol. 48, no. 10, pp. 1065-1070, 2001.

[10] B. M. Bugbee, "An Implantable Stimulation for Selective Stimulation of Nerves," PhD thesis, University College London, , 2000.

[11] O. Macherey, A. van Wieringen, R. P. Carlyon, J. M. Deeks, and J. Wouters, "Asymmetric pulses in cochlear implants: Effects of pulse shape, polarity, and rate," J. Assoc. for Res. in Otolaryngol., vol. 7, no. 3 , pp. 253-266, 2006.

[12] T. G. Constandinou, J. Georgiou, C. C. Doumanidis, and C. Toumazou, "Towards an implantable vestibular prosthesis: The surgical challenges," in Proc. IEEE EMBS Conf. on Neural Eng., 2007, pp. 40-43.

[13] M. Illic, D. Vasiljevic, and D. B. Popovic, "A programmable electronic stimulator for FES systems," IEEE Trans. Rehab. Eng., vol. 2, no. 4, pp. 234-239, Dec. 1994.

[14] T. Stieglitz, T. Matal, and M. Staemmler, "A modular multichannel stimulator for arbitrary shaped current pulses for experimental and clinical use in FES," in Proc. IEEE Int. Conf. of EMBS, 1997, pp. $1777-1780$.

[15] G. Gudnason, E. Bruun, and M. Haugland, "An implantable mixed analog/digital neural stimulator circuit," in Proc. IEEE Int. Symp. on Circuits Syst., 1999, vol. 5, pp. 375-378.

[16] K. Arabi and M. A. Sawan, "Electronic design of a multichannel programmable implant for neuromuscular electrical stimulation," IEEE Trans. Rehab. Eng., vol. 7, no. 2, pp. 204-214, 1999.

[17] C.-W. Chang, W.-Y. Chung, C.-C. Chuang, R.-L. Wei, and J.-X. Dai, "A novel system design for implantable stimulator application," in Proc. IEEE Int. Conf. EMBS, 2004, pp. 4107-4110.

[18] C.-C. Wang, T.-J. Lee, Y.-T. Hsiao, U. F. Chio, C.-C. Huang, J.-J. J. Chin, and Y.-H. Hsueh, "A multiparameter implantable microstimulator SOC," IEEE Trans. Very Large Scale Integr. (VLSI) Syst., vol. 13, no. 12, pp. 1399-1402, Dec. 2005. 
[19] M. Sivaprakasam, W. Liu, M. S. Humayun, and J. D. Weiland, "An arbitrary waveform stimulus circuit for visual prostheses using a lowarea multibias DAC," IEEE J. Solid-State Circuits, vol. 40, no. 3, pp. 763-771, Mar. 2005.

[20] J. Couloumbe, M. Sawan, and J.-F. Gervais, "A highly flexible system for microstimulation of the visual cortex: Design and implementation," IEEE Trans. Biomed. Circuits Syst., vol. 1, no. 4, pp. 258-269, Dec. 2007.

[21] M. Ortmanns, A. Rocke, M. Gehrke, and H.-J. Tiedtke, "A 232-channel epiretinal stimulator ASIC," IEEE J. Solid-State Circuits, vol. 42, no. 12, pp. 2946-2959, Dec. 2007.

[22] A. Eftekhar, T. G. Constandinou, I. F. Triantis, C. Toumazou, and E. M. Drakakis, "Towards a reconfigurable sense-and-stimulate neural interface generating biphasic interleaved stimulus," in Proc. IEEE EMBS Conf. Neural Eng., 2007, vol. 3, pp. 438-441.

[23] D. B. Koch, M. Downing, M. J. Osberger, and L. Litvak, "Using current steering to increase spectral resolution in CII and HiRes $90 \mathrm{~K}$ users," AAS Ear and Hearing, vol. 28, no. 2, 2007.

[24] B. S. Wilson, C. C. Finley, D. T. Lawson, R. D. Wolford, and M. Zerbi, "Design and evaluation of a CIS processing strategy for multichannel cochlear implants," J. Rehab. Res. Develop., vol. 30, no. 1, pp. 110-116, 1993.

[25] J. Georgiou and C. Toumazou, "A $126 \mu \mathrm{W}$ cochlear chip for a totally implantable system," IEEE J. Solid-State Circuits, vol. 40, no. 2, pp. 430-443, Feb. 2005.

[26] S. C. DeMarco, W. Liu, P. R. Singh, G. Lazzi, M. S. Humayun, and J. D. Weiland, "An arbitrary waveform stimulus circuit for visual prostheses using a low-area multibias DAC," IEEE J. Solid-State Circuits, vol. 38, no. 10, pp. 1679-1790, Oct. 2003.

[27] O. Omeni and C. Toumazou, "A CMOS micro-power wideband data/ power transfer system for biomedical implants," in Proc. IEEE Int. Symp. on Circuits Syst., 2003, vol. 5, pp. 61-65.

[28] C.-C. Wang, Y.-H. Hsueh, Y.-T. Hsiao, U. F. Chiot, C.-C. Huang, and P.-L. Liu, "An implantable neural interface micro-stimulator chip with external controllability," in Proc. IEEE Asia-Pacific Conf. on Adv. Syst. Integr. Circuits, 2004, pp. 356-359.

[29] S. J.-J. Sit and R. Sarpeshkar, "A low-power blocking-capacitor-free charge-balanced electrode-stimulator chip with less than 6 nA DC error for 1-mA full-scale stimulation," IEEE Trans. Biomed. Circuits Syst., vol. 1, no. 3, pp. 172-183, Sep. 2007.

[30] X. Fang, J. Wills, J. Granacki, J. LaCoss, A. Arakeliana, and J. Weiland, "Novel charge-metering stimulus amplifier for biomimetic implantable prosthesis," in Proc. IEEE Int. Symp. on Circuits Syst., 2007, pp. 569-572.

[31] E. K. F. Lee and A. Lam, "A matching technique for biphasic stimulation pulse," in Proc. IEEE Int. Symp. on Circuits Syst., 2007, pp. $817-820$.

[32] M. Ortmanns, N. Unger, A. Rocke, M. Gehrke, and H. J. Tietdke, “A $0.1 \mathrm{~mm}$, digitally programmable nerve stimulation pad cell with highvoltage capability for a retinal implant," in Proc. IEEE Int. Solid-State Circuits Conf., 2006, pp. 89-98.

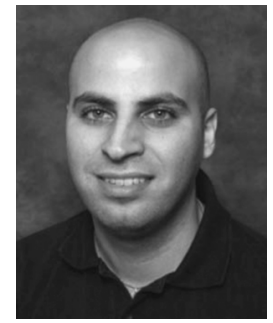

Timothy G. Constandinou (M'98) received the B.Eng. degree in electrical and electronic engineering (first class hons.) in 2001 and the Ph.D. degree in electronic engineering from Imperial College of Science, Technology and Medicine, in 2005.

He is currently based at the Institute of Biomedical Engineering at Imperial, where he is Research Officer for the Bionics research group. He also holds a position of research Fellow at the University of Cyprus. His research interests include ultra low power circuits and systems for biomedical and biologically-inspired applications. These include implantable neuroprosthetic devices, body-worn instrumentation, integrated smart sensors and vision systems.

Dr. Constandinou is a member of the IEEE Circuits and Systems Society, Sensory Systems, and BioCAS Technical Committees.

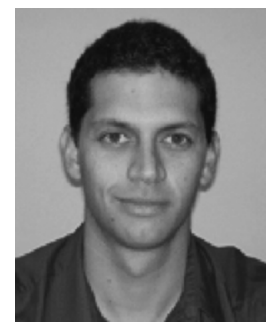

Julius Georgiou (M'98) received the M.Eng. degree in electrical and electronic engineering and Ph.D. degree in biomedical electronics from Imperial College London in 1998 and 2003, respectively.

During the last two years of his Ph.D. he was heavily involved in a technology startup company, Toumaz Technology, as Head of Micropower Design. In 2004 he joined the Johns Hopkins University as a Postdoctoral Fellow, before joining the University of Cyprus in 2005. His main area of expertise is in ultra low power circuit techniques and has applied them to a range of applications spanning from biomedical implants to defense systems.

Dr. Georgiou is a member of the IEEE Circuits and Systems Society, the BioCAS Technical Committee, as well as a member of the IEEE Circuits and Systems Society Analog Signal Processing Technical Committee.

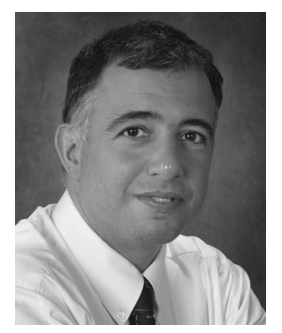

Christofer Toumazou (M'87-SM'99-F'01) is a Professor of Circuit Design, Founder and Executive Director of the Institute of Biomedical Engineering at Imperial College London, London, UK. Professor Toumazou has made outstanding contributions to the fields of low power analogue circuit design and current mode circuits and systems for biomedical and wireless applications. Through his extensive record of research, he has invented innovative electronic devices ranging from dual mode cellular phones to ultra-low power devices for both medical diagnosis and therapy. He has published over 320 research papers in the field of RF and low power electronics and. He holds 23 patents in the field, many of which are now fully granted PCT. He is the founder of four technology based companies with applications spanning ultra low-power mobile technology and wireless vital sign monitors (Toumaz Technology Ltd, U.K.), biomedical devices (Applied Bionics PTE, Singapore), Digital Audio Broadcasting (Future-Waves Pte Taiwan) and DNA Sequencing (DNA Electronics Ltd, UK). These companies employ over 50 RF low power engineers worldwide many of whom are Professor Toumazou's ex graduate students. Professor Toumazou was invited to deliver the 2003 Royal Society Clifford Patterson Prize Lecture, entitled "The Bionic Man", for which he was awarded The Royal Society Clifford Patterson bronze medal. He was recently awarded the IEEE CAS Society Education Award for pioneering contributions to telecommunications and biomedical circuits and systems, and the Silver Medal from the Royal Academy of Engineering for his outstanding personal contributions to British engineering. 